\title{
A BOUNDARY VALUE PROBLEM FOR BELTRAMI DIFFERENTIAL EQUATION
}

\author{
KEIICHI SHIBATA \\ Department of Mathematics, Okayama University of Science \\ 1-1 Ridai-cho Okayama, 700, Japan \\ E-mail:tym0510@ousic.ous.ac.jp
}

\begin{abstract}
Solutions to Beltrami differential equation with prescribed boundary correspondence in some plane domains are given.

1. Introduction. It belongs to quite a classical problem which had indeed been discussed first by Gauss in the era of nineteenth century, to introduce isothermal coordinates into a given differential-geometric surface:it was formulated afterwards by E. Beltrami in a complex version, namely it reduced to find the vanishing point for a given differential
\end{abstract} operator

$$
\frac{\partial}{\partial \bar{z}}-\mu(z) \frac{\partial}{\partial z}
$$

where $z$ is a complex variable, $\bar{z}$ its conjugate and $\mu$ a complex-valued function with some smoothness properties subject to the inequality $|\mu(z)|<1$. The global pursuit of the above problem could not necessarily circumvent the serious difficulties accompanied with topological nature of the surface itself. For such reason, perhaps, the development of this line of arguments seems to have aimed at relaxation of the smoothness assumption to be imposed on the coefficient $\mu(z)$ exclusively for the region without boundary, especially for $\widehat{\mathbb{C}}$.

In the present study we will attack the question how to solve the Beltrami equation in some plane domain in relation to a prescribed 1-dimensionally quasiconformal boundary homeomorphism.

Our method consists in a simple application of Green's formula as well as C. Neumann's series in the theory of integral equations.

2. Heuristics and integral formula for the mapping function. Let $R$ be a Riemann surface and let $z$ a local coordinate associated with a coordinate neighbourhood

1991 Mathematics Subject Classification: Primary 30C62; Secondary 35F15.

The paper is in final form and no version of it will be published elsewhere. 
$V$ of $R$. Gerstenhaber-Rauch [3] utilized the coordinate change

$$
\zeta=z+\varepsilon h(z)
$$

for the deformation of complex analytic structure of $V$ in $R$ in their own variational problem, where $h(z)$ is an arbitrary function of class $\mathrm{C}^{1, \alpha}$ supported on Clo $V$ and $\varepsilon$ is any sufficiently small complex constant. For the purpose of arguments that follow we are free to alter the above situation in such a way that we only consider the mapping

$$
w=f(z)=z+\varepsilon h(z)
$$

of the unit open disk $U_{z}$ in the $z$-plane instead of the coordinate change in $V$ and that the support of $h(z)$ agrees with Clo $U_{z}$ itself; the existence of such $h(z)$ will be guaranteed, e.g. in L. Schwartz [5], p.15, even in the $\mathrm{C}^{\infty}$ class. Smallness of $|\varepsilon|$ relative to the $h(z)$ fixed in advance results that $f(z)$ is a homeomorphism of Clo $U_{z}$ onto another unit closed disk Clo $U_{w}$ in the $w$-plane, which is quasiconformal in $U_{z}$ and stabilizes the circumference pointwise. For later convenience we shall refer to this class of mappings by $\mathfrak{H}$. In accordance with the customary notations in quasiconformal mapping theory let us write $p=\partial f / \partial z, q=\partial f / \partial \bar{z}$ for the complex derivatives of $f(z)$ belonging to $\mathfrak{H}$, in terms of which the Beltrami coefficient is defined by putting $\mu=q / p$ so far as $p \neq 0$. For the mapping (2) we see of course that $p=1+\varepsilon h_{z}$ and $q=\varepsilon h_{\bar{z}}$.

Note that any $f(z)$ of $\mathfrak{H}$ is conformal outside of $U_{z}$, i.e. the Beltrami coefficient $\mu$ of this $f(z)$ approaches 0 as $z$ tends to $\partial U_{z}$ from $\operatorname{Int} U_{z}$.

Meanwhile the following very useful expression representing a kind of complex versions of Green's theorem should priorily be attributed to M.D. Pompeiu [4].

LEMma 1. Let $D$ denote an arbitrary domain in the z-plane bounded by a finite number of disjoint regular analytic Jordan curves and let $f(z)$ a complex-valued function continuous on Clo D, while it is of class $\mathrm{C}^{1, \alpha}(0<\alpha \leq 1)$ in D. Then there holds the identity

$$
f(z)=\frac{1}{2 \pi i} \oint_{\partial D} \frac{f(\zeta)}{\zeta-z} d \zeta+\frac{1}{2 \pi i} \iint_{D} \frac{q(\zeta)}{\zeta-z} d \zeta d \bar{\zeta} \quad(z \in D) .
$$

Proof. We fix an arbitrary point $z$ in the interior of $D$. For any $\varepsilon>0$ there is some $\delta>0$ such that $|\zeta-z|<\delta$ implies $|f(\zeta)-f(z)|<\varepsilon$. With a positive number $\delta^{\prime}$ not exceeding $\delta$ one deletes from $D$ a closed disk $C=\left\{\zeta|| \zeta-z \mid \leq \delta^{\prime}\right\}$ comprised in $D$ to obtain a subdomain $D^{*}$ of $D$. Then Green's theorem yields

$$
\begin{aligned}
& \oint_{\partial D} \frac{f(\zeta)-f(z)}{\zeta-z} d \zeta-\oint_{\partial C} \frac{f(\zeta)-f(z)}{\zeta-z} d \zeta \\
& =-\iint_{D^{*}} \frac{\partial}{\partial \bar{\zeta}}\left(\frac{f(\zeta)-f(z)}{\zeta-z}\right) d \zeta d \bar{\zeta}=-\iint_{D^{*}} \frac{q(\zeta)}{\zeta-z} d \zeta d \bar{\zeta}
\end{aligned}
$$

Hence

$$
\left|\frac{1}{2 \pi i} \oint_{\partial D} \frac{f(\zeta)-f(z)}{\zeta-z} d \zeta+\frac{1}{2 \pi i} \iint_{D^{*}} \frac{q(\zeta)}{\zeta-z} d \zeta d \bar{\zeta}\right| \leq \frac{1}{2 \pi} \oint_{\partial C}\left|\frac{f(\zeta)-f(z)}{\zeta-z}\right||d \zeta|<\varepsilon .
$$

Letting $\delta$ tend to zero, we have the conclusion. 
We wish to remark that it is possible to generalize and modify the class $\mathfrak{H}$, to which Lemma 1 applies in the following way.

Consider the family $\mathfrak{E}$ of homeomorphisms $f(z)$ of Clo $D$ such that

(a) $f(z)$ is continuous on $z$-plane $\mathbb{C}$;

(b) $f(z)$ is quasiconformal in $D$;

(c) the Beltrami coefficient $\mu(z)$ of $f(z)$ approaches 0 as $z$ tends to $\partial D$ from Int $D$;

(d) $f(z)=z$ on $\partial D$;

(e) $f(z)$ is conformal outside of Clo $D$.

Under these conditions we see easily that

$$
\frac{1}{2 \pi i} \oint_{\partial D} \frac{f(\zeta)}{\zeta-z} d \zeta=\frac{1}{2 \pi i} \oint_{\partial D} \frac{\zeta}{\zeta-z} d \zeta=z .
$$

Hence

Corollary. For every homeomorphism $f(z)$ belonging to $\mathfrak{E}$ it holds that

$$
f(z)=z+\frac{1}{2 \pi i} \iint_{D} \frac{q(\zeta)}{\zeta-z} d \zeta d \bar{\zeta}, \quad \text { where } \quad q=\partial f / \partial \bar{z}
$$

3. Basic lemma. It will be convenient for later use to introduce the Hilbert transform of a complex function $g(z)$ which is of class $\operatorname{Lip} \alpha$ with compact support on $\mathbb{C}$. It takes the following form in our situation whose existence is guaranteed by the Hölder continuity imposed on $g$ : the integrations should be evaluated over the whole $\zeta:(=\xi+i \eta)$-plane $(\xi, \eta \in \mathbb{R})$.

DeFinition 1. (pre-Hilbert transform)

$$
(P g)(z)=\frac{1}{2 \pi i} \iint \frac{g(\zeta)}{\zeta-z} d \zeta d \bar{\zeta}
$$

DeFinition 2. (Hilbert transform)

$$
(T g)(z)=\frac{1}{2 \pi i} \iint \frac{g(\zeta)}{(\zeta-z)^{2}} d \zeta d \bar{\zeta}
$$

If $f(z)$ is, in particular, of the family $\mathfrak{E}$, Corollary to Lemma 1 allows us to write

$$
f(z)=z+(P q)(z)
$$

where $q=\varepsilon h_{\bar{z}}$. Differentiation of the relation (5) with respect to $z$ yields that

$$
p=1+T q,
$$

which will be utilized in the next section.

Our observation so far was based upon the fact that every element of $\mathfrak{E}$ admits the expression (4). Now we assert that the converse is also true. To this end, suppose we are given a Beltrami coefficient $\mu$ which is bounded by a positive constant $k$ smaller than 1 , 
supported by Clo $D$, and belongs to the $\mathrm{C}^{1, \alpha}$ on the whole $z$-plane $\mathbb{C}$. Then multiplication of $\mu$ with (6) provides the equation

$$
q=\mu+\mu T q
$$

Our next task is to look for the unknown $q$ that suits (7). The last question can be solved, e.g. in terms of the Neumann series containing the Hilbert transform of $\mu$

$$
q=\mu+\mu T \mu+\mu T \mu T \mu+\ldots,
$$

which turns out to converge in some normed space, and to which the given $\mu$ belongs.

Re mark 1. In order to verify the above assertion one refers to, e.g. Ahlfors [1], pp. 3 - 7. Indeed let $\mathfrak{M}$ denote the set of complex-valued function $\mu(z)$ satisfying the following conditions:

(A) $\mu$ is defined on the whole $z$-plane $\mathbb{C}$;

(B) $\operatorname{supp} \mu$ coincides with Clo $D$;

(C) $\mu \in \operatorname{Lip} \alpha:(0<\alpha \leq 1)$;

(D) $\mathfrak{M}$ is endowed with some norm: to be precise every $\mu$ of $\mathfrak{M}$ fulfills the inequality

$$
\left|\mu\left(z_{1}\right)-\mu\left(z_{2}\right)\right| \leq A\left|z_{1}-z_{2}\right|^{\alpha}
$$

for an arbitrary pair of points $z_{1}, z_{2} \in \mathbb{C}$ with some constant $A$. One writes $|\mu|_{\alpha}$ for the maximal value of such Lipschitz constant $A$. Further we set $\langle\mu\rangle_{\alpha}=\max \left\{\|\mu\|,|\mu|_{\alpha}\right\}$, $\|\mu\|$ being the $\mathrm{L}^{2}$ norm of $\mu$.

$\mathfrak{M}$ is complete with this norm $\langle\mu\rangle_{\alpha}$. Then if $|\mu|<1 / 8 C_{1}$ with

$$
C_{1}=\sup _{\mu \in \mathfrak{M}} \frac{|T \mu(1)-T \mu(-1)|}{2^{\alpha}|\mu|_{\alpha}},
$$

then it holds that $|T \mu|<1$. So the series (8) converges in the space $\mathfrak{M}$, and $q$ belongs again to $\mathfrak{M}$.

Thus we have proved

Proposition 1. Suppose that $\mu$ belonging to $\mathfrak{M}$ fulfills the assumption $|\mu|<1 / 8 C_{1}$. Then under the condition that $\lim _{z \rightarrow \partial D} \mu(z)=0$, there exists a unique quasiconformal map $f(z)$ which has the following properties: for any point $z$ in $D$ it holds that

$$
\frac{\partial f(z)}{\partial \bar{z}}=\mu(z) \frac{\partial f(z)}{\partial z}
$$

and if $z$ is on $\partial D$, we have $f(z)=z$.

Removal of the subsidiary condition $|\mu| \ll 1$ can be carried out by means of a kind of superposition.

Putting $\zeta=f(z)$ with the mapping $f(z)$ already obtained in Proposition 1 one adopts the $\zeta$ as the the new independent variable. If one chooses a sufficiently small Beltrami coefficient $\mu_{1}(\zeta)$ satisfying the boundary condition $\mu_{1}(\zeta) \rightarrow 0$ as $\mu_{1}(\zeta) \rightarrow \partial D$, there is a unique fucntion $f_{1}(\zeta)$, such that if $z$ belongs to the interior of $D$, then $\partial f_{1}(\zeta) / \partial \bar{z}=$ $\mu_{1}(\zeta) \partial f_{1}(\zeta) / \partial \zeta$, while for any $z$ on $\partial D$ one has $f_{1}(\zeta)=\zeta$.

It is evident that the composite map $f_{1} \circ f(z)$ fulfills the condition (a),(b) in the preceding section. For the given $\mu(z)$ it is possible to take a finite sequence of Beltrami 
coefficients $\mu_{1}, \mu_{2}, \ldots, \mu_{N}$, each of which is subject to the subsidiary condition imposed in Proposition 1. Therefore we have

TheOREM 1. Suppose we are given a Beltrami coefficient $\mu(z)$ in the domain $D$. Under the condition that $\mu(z)$ tends to zero as $z$ approaches $\partial D$, there exists a unique quasiconformal homeomorphism $f^{*}(z)$ of $D$, such that

(a) for any $z$ in $D, f^{*}(z)$ satisfies the Beltrami equation $f_{\bar{z}}^{*}(z)=\mu(z) f_{z}^{*}(z)$;

(b) on the boundary $\partial D, f^{*}(z)$ fixes all the boundary points.

4. Main theorem. Roughly speaking, the composite map of any two quasiconformal mappings is also quasiconfomal, restricted to the local consideration. But the most exact form of this fact is formulated, e.g. in Ahlfors [2], pp. 8-10.

Suppose, for instance, we are given a function $\Phi(z)$ of $\mathrm{C}^{1, \alpha}(\mathbb{C}, \mathbb{C})$, which realizes a homeomorphism of Clo $D$, which is quasiconformal but nowhere conformal in $D$ and has the boundary value $\varphi(z)$. Let the Beltrami coefficient of $\Phi(z)$ be $\mu(z)$, namely, $\Phi(z)$ satisfies the equation

$$
\Phi_{\bar{z}}=\mu(z) \Phi_{z} \quad \text { in } D .
$$

On the other hand there be assigned an arbitrary Beltrami coefficient $\nu(z)$ in $D$ which is of class $\operatorname{Lip} \alpha(\operatorname{Clo} D, \mathbb{C})$, such that $\nu(z)=\mu(z)$ on $\partial D$. Then Theorem 1 enables us to define the homeomorphism $\Omega(z)$ of Clo $D$ which is quasiconformal in $D$ with the Beltrami coefficient

$$
\lambda=\frac{\Phi_{z}}{\Phi_{\bar{z}}} \cdot \frac{\nu-\mu}{1-\bar{\mu} \nu}
$$

and which fixes the boundary $\partial D$ pointwise. If we compose $\Omega$ with the prescribed $\Phi$ to define the new one $\Psi(z)$ in such a way that $\Psi=\Omega \circ \Phi$, then $\Psi(z)$ provides the homeomorphism Clo $D$ which is quasiconformal with the prescribed Beltrami coefficient $\nu(z)$ (cf. Ahlfors [2], (10), p.9) and has the same boundary value $\varphi$ as the given one of $\Phi(z)$. Thus we have proved

THEOREM 2 Given a homeomorphism belonging to $\mathrm{C}^{1, \alpha}(\mathbb{C}, \mathbb{C})$ which is quasiconformal in $D$ with the boundary value $\varphi(z)$. Then, for any complex-valued function $\nu(z)$ of $\operatorname{Lip} \alpha$ and satisfying $|\nu(z)| \leq k<1$ ( $k=$ const), there exists a unique solution to the Beltrami equation

$$
\frac{\partial w}{\partial \bar{z}}=\nu(z) \frac{\partial w}{\partial z},
$$

with the assigned boundary value $\varphi(z)$.

\section{References}

[1] L.V. Ahlfors, Conformality with respect to Riemannian metrics, Ann. Acad. Sci. Fenn. A I 206 (1955), 1-22.

[2] - Lectures on quasiconformal mappings, Van Nostrand, Princeton, Toronto, New York, London 1966.

[3] M. Gerstenhaber and H.E. Rauch, On quasiconformal mappings I, Proc. Nat. Acad. Sci. U.S.A. 40 (1954), 808-812. 
[4] M. D. Pompeiu, Sur une classe de fonctions d'une variable complexe et certaines équations intégrales, Rend. Circ. Mat. Palermo 35 (1913), 277-281.

[5] L. Schwartz, Théorie des distributions, tome I, Hermann, Paris 1950. 